\title{
Readmission destination and risk of mortality after major surgery: an observational cohort study
}

\author{
Benjamin S Brooke, MD, Philip P Goodney, MD, Prof Larry W Kraiss, MD, Daniel J Gottlieb, \\ MS, Prof Matthew H Samore, MD, and Prof Samuel R G Finlayson, MD \\ Department of Surgery (B S Brooke MD, Prof L W Kraiss MD, Prof S R G Finlayson MD) and \\ Division of Epidemiology (Prof M H Samore MD), University of Utah School of Medicine, Salt \\ Lake City, UT, USA; IDEAS Center, VA Salt Lake City Health Care System, Salt Lake City, UT, \\ USA (B S Brooke, Prof M H Samore); Section of Vascular Surgery, Dartmouth-Hitchcock Medical \\ Center, Lebanon, NH, USA (PP Goodney MD); and The Dartmouth Institute for Health Policy and \\ Clinical Practice, Hanover, NH, USA (D J Gottlieb MS)
}

\section{Summary}

Background-Hospital readmissions are common after major surgery, although it is unknown whether patients achieve improved outcomes when they are readmitted to, and receive care at, the index hospital where their surgical procedure was done. We examined the association between readmission destination and mortality risk in the USA in Medicare beneficiaries after a range of common operations.

Methods-By use of claims data from Medicare beneficiaries in the USA between Jan 1, 2001, and Nov 15, 2011, we assessed patients who needed hospital readmission within 30 days after open abdominal aortic aneurysm repair, infrainguinal arterial bypass, aortobifemoral bypass, coronary artery bypass surgery, oesophagectomy, colectomy, pancreatectomy, cholecystectomy, ventral hernia repair, craniotomy, hip replacement, or knee replacement. We used logistic regression models incorporating inverse probability weighting and instrumental variable analysis to measure associations between readmission destination (index $v s$ non-index hospital) and risk of 90 day mortality for patients who underwent surgery who needed hospital readmission.

Findings-9 440503 patients underwent one of 12 major operations, and the number of patients readmitted or transferred back to the index hospital where their operation was done varied from $186336(65.8 \%)$ of 283131 patients who were readmitted after coronary artery bypass grafting, to $142142(83.2 \%)$ of 170789 patients who were readmitted after colectomy. Readmission was more likely to be to the index hospital than to a non-index hospital if the readmission was for a surgical

Correspondence to: Dr Benjamin S Brooke, Department of Surgery, University of Utah School of Medicine, Salt Lake City, UT 84132, USA benjamin.brooke@hsc.utah.edu.

For the American Community survey data see http://www.census.gov/acs/www/data_documentation/data_main/

For the Hospital Compare website see http://www.medicare.gov/hospitalcompare/search.html

See Online for appendix

Contributors

BSB, PPG, MHS, and SRGF conceived and designed the study. DJG did the analyses and, with BSB, PPG, LWK, MHS, and SRGF assisted with the study design and analysis. BSB drafted the report and revised it with contributions from all authors.

Declaration of interests

We declare no competing interests. 
complication (189 384 [23\%] of 834070 patients readmitted to index hospital vs 36792 [13\%] of 276976 patients readmitted non-index hospital, $\mathrm{p}<0 \cdot 0001$ ). Readmission to the index hospital was associated with a $26 \%$ lower risk of 90 day mortality than was readmission to a non-index hospital, with inverse probability weighting used to control for selection bias (odds ratio [OR] $0 \cdot 74,95 \%$ CI $0.66-0.83)$. This effect was significant $(\mathrm{p}<0.0001)$ for all procedures in inverse probability-weighted models, and was largest for patients who were readmitted after pancreatectomy (OR 0.56, 95\% CI 0.45-0.69) and aortobifemoral bypass (OR 0.69, 95\% CI 0.610.77). By use of hospital-level variation among regional index hospital readmission rates as an instrument, instrumental variable analysis showed that the patients with the highest probability of returning to the index hospital had $8 \%$ lower risk of mortality (OR $0.9295 \%$ CI $0.91-0.94$ ) than did patients who were less likely to be readmitted to the index hospital.

Interpretation-In the USA, patients who are readmitted to hospital after various major operations consistently achieve improved survival if they return to the hospital where their surgery took place. These findings might have important implications for cost-effectiveness-driven regional centralisation of surgical care.

\section{Introduction}

Identification of metrics for quality of surgical care has become a major priority for healthcare providers, patients, those paying for health care, and policy makers in many countries. So far, the main focus has been on perioperative measures of surgical quality, including structural characteristics of hospitals where surgery takes place and measures of the perioperative process within hospitals that lead to the best postoperative outcomes. ${ }^{1,2}$ Hospitals that provide high-quality surgical care are often labelled as so-called centres of excellence, and trends have emerged in support of cost-effectiveness-driven regional centralisation for complex and major surgery. ${ }^{3,4}$ These changes were based on reports ${ }^{5-9}$ showing that hospitals with certain characteristics-eg, high operative volume or specialty care pathways-are better able to manage patients undergoing complex surgery and resulting complications, leading to reduced rates of risk-adjusted mortality and readmission.

However, after patients have been discharged from hospital following major surgery, the factors that are associated with improved outcomes are unclear. This challenge is relevant in view of the fact that a substantial proportion of complications and deaths within 90 days after major surgery occur after patients have been discharged from hospital, ${ }^{10}$ and up to $25 \%$ of patients will need readmission. ${ }^{11,12}$ Because the need for hospital readmission after major surgery is associated with significantly increased risk of mortality, ${ }^{13,14}$ metrics of quality for surgical readmission need to be defined.

Maintenance of continuity of care with the same health-care institutions and providers is an established metric of quality for patients treated for chronic medical conditions. ${ }^{15,16} \mathrm{We}$ postulated that this quality metric would also apply to patients who were readmitted to hospital after major surgery, who we suspected would achieve improved outcomes if they returned to the hospitals where their operation took place (ie, the index hospital). We aimed to assess readmission destination and risk-adjusted 90 day mortality estimates for fee-for- 
service Medicare beneficiaries in the USA who were readmitted to hospital within 30 days after 12 common operations across five surgical disciplines.

\section{Methods}

\section{Data sources and study population}

We used the Centers of Medicare and Medicaid Services Provider Analysis and Review database to study patients who were readmitted to hospital within 30 days after undergoing one of 12 major surgical procedures at acute care and critical access hospitals between Jan 1, 2001, and Nov 15, 2011. We used International Classification of Diseases 9th Revision (ICD-9) procedure codes to identify these procedures from the Part A Medicare claims dataset: open abdominal aortic aneurysm repair $(38.34,38.36,38.44,38.64,39.25$, and 39.52); aortobifemoral bypass (39.25); infrainguinal arterial bypass $(39.29,38.88,38.48$, and 38.38); coronary artery bypass grafting (36.10-36.19); cholecystectomy (51.21-51.24); colectomy (45.7-45.79, 45.8); pancreatectomy (52.70, 52.51-52.53, 52.59, 55.26); oesophagectomy $(42.4,42.40-42.42)$; craniotomy $(01.20-01.28,01.30-01.32,01.39,01.50-$ 01.53, 01.59), ventral hernia repair $(53.51,53.61,53.62)$; hip replacement (81.51); and knee replacement (81.54). In our analysis, we included all patients who made use of a fee-forservice, non-health maintenance organisation (HMO) payment model, who were aged younger than 99 years and underwent one of these 12 operations during the time period.

To be included in the cohort for analysis, all patients needed to be readmitted within 30 days of discharge after one of the 12 index surgical procedures. Only the first unplanned readmission during the first 30 postoperative days was examined. If patients were transferred to another institution during the postoperative period, the 30 day window started after discharge from the other institution.

\section{Readmission destination}

Our main exposure variable was readmission destination, defined by whether patients were readmitted to the index hospital where the surgery took place or a different, non-index, hospital. The exposure group included patients who were readmitted to outside hospitals but then transferred to the index hospital within $24 \mathrm{~h}$. The reference group included all patients who were readmitted to, and cared for at, hospitals other than the index hospital. The definition of index versus non-index hospital admission was specific for each patient, which allowed for cross-classification of data. We used hospital identification numbers from the Centers of Medicare and Medicaid Services to categorise index and readmission hospitals.

Additionally, we assessed whether having the same surgical provider was associated with both index and readmission hospitalisations by use of Berenson-Eggers Type of Service (BETOS) codes. BETOS codes are associated with services that health-care providers perform for Medicare beneficiaries and submit for billing, including evaluation and management and medical procedures. We included billing claims from the operative surgeon, and nurse practitioners or physician assistants who might have been directly involved in patient care, and compared these claims with the provider billing claims for evaluation and management and medical procedures during the period of readmission. 


\section{Descriptive variables}

We assessed differences in readmission destination by patient characteristics at several levels. Baseline demographic variables included age, sex, ethnic origin, disability, and Medicaid eligibility at the time of index hospital admission. We obtained data for education and median income from the American Community survey data (2006 to 2010) and used them to measure patient socioeconomic status. We assessed patients' comorbidities with a Charlson index (Romano adaptation) ${ }^{17}$ score generated from the diagnosis at index hospital admission and procedure codes. We calibrated the Charlson index weights to a surgical hip fracture cohort that has previously been validated in various populations who had surgery. ${ }^{12,18}$ We coded each patient's ambulatory status with CPT codes within claims and carrier files for walkers, wheelchairs, and related accessories 2 years before and up to 30 days after the index surgery. Finally, we categorised the patient's discharge destination after their index hospital admission for surgery (home, skilled nursing facilities, rehabilitation facility, home with home-care services, or other).

We defined readmissions by whether they were elective, urgent, or emergent, and whether the readmission was to manage a medical complication (eg, heart failure or peneumonia) or a surgical complication (ie, a complication requiring a procedure at the time of readmission such as wound debridement). We based this distinction on the medical and surgical diagnosis-related groups assigned to patients at the time of readmission. We categorised the source of admission to the hospital at the time of readmission as being from an emergency department, outpatient clinic, transfer from another hospital facility, or another source.

We characterised the hospitals patients were readmitted to in several ways. We assessed overall hospital volume of admissions and procedure-specific volumes by hospital. We measured admission volumes across all years combined and with biannual values smoothed by a moving average. For all 12 procedures, the top $10 \%$ of hospitals ranked by volume accounted for roughly $50 \%$ of operations, and we used this cutoff point to define hospitals with the highest volumes of procedures. We categorised the teaching status of hospitals (non-teaching, minor teaching, and major teaching) based on the ratio of junior doctors to beds, as identified by the 2010 American Hospital Association files, and whether hospitals were members of the Council of Teaching Hospitals. Additionally, we assessed the number of hospital beds, intensive-care unit beds, physician staffing (full-time equivalents), and nurse-to-patient ratios using the year-specific American Hospital Association files. We assessed hospital compliance with established process measures derived from the Surgical Care Improvement Program (SCIP), using the 2012 Hospital Compare website. We chose SCIP-9 (compliance with removal of urinary catheters by postoperative day 2) because this measure had the most variability and discriminatory potential between hospitals in the USA.

We used the University of Washington Rural-Urban Commuting Area (RUCA) Version 2 codes to categorise the locations of hospitals and patients' homes with zip codes, aggregated to four levels: urban, suburban, large town, or rural. We calculated the distance from the patient's home to the hospital as a straight-line distance measured in miles. Finally, we assessed whether patients underwent their index surgical procedure within the US hospital referral region associated with their home address. 


\section{Outcomes}

The main outcome measure was 90 day all-cause mortality, beginning from the day of hospital readmission. We obtained dates of death from the Medicare Vital Status file. We also assessed in-hospital mortality during the period of hospital readmission.

\section{Statistical analysis}

We used two methods to assess the primary outcome measure: inverse probability weighting and an instrumental variable analysis. ${ }^{19}$ We did all analyses with SAS version 9.3.

To address confounding by measured covariates, we used logistic regression incorporating inverse probability weighting, a type of propensity score analysis. ${ }^{20} \mathrm{We}$ first calculated descriptive statistics for the predictor variables measured within cohorts of patients undergoing each operation using bivariate comparisons ( $\chi^{2}$ and ANOVA). We then used multiple logistic regression to calculate the probability of a patient being readmitted to the index hospital based on 74 covariates (appendix). The weights for each patient were defined as the inverse of the estimated probabilities for being readmitted to the index hospital. After we weighted patients, we ran logistic regression models for each of the 12 surgical procedures clustered by hospital size (ie, small, medium, and large) to estimate odds ratios (ORs) for mortality. Model convergence was not possible when we attempted clustering at the level of individual hospitals because many US hospitals had few patients in each procedure group. We also did risk adjustment for all 12 procedures using logistic regression models without inverse probability weighting and without clustering.

We calculated $\mathrm{p}$ values for comparisons between index and non-index hospitals using $\boldsymbol{t}$ tests for means and $\chi^{2}$ tests for proportions. We made categorical comparisons with $\chi^{2}$ tests or ANOVA, as appropriate.

To address potential unmeasured bias, we did instrumental variable analysis with hospitallevel variation in regional index hospital 30 day readmission rates as an instrument. Hospitals within each hospital referral region were divided into quartiles based on index readmission rates after each operation, and we then compared groups of patients at hospitals that differed in terms of high and low probability of being readmitted to the index hospital (appendix). This type of geographic instrumental variable behaves like a natural random assignment of patients who underwent surgery to regional exposure groups that differ in likelihood of returning to the index hospital at the time of readmission. ${ }^{19}$ We estimated riskadjusted ORs for 90 day mortality for all 12 procedures with comparisons between hospitals at low and high risk of index readmission compared with non-index readmission.

To investigate whether having the same surgical providers during both index hospital admission and readmission was associated with a survival benefit, we calculated adjusted inhospital and 90 day mortality based on whether patients were readmitted to non-index hospitals, index hospitals with different providers, or index hospitals with the same providers. We did incremental $\mathrm{R}^{2}$ analysis to calculate the fraction of the variance accounted for in the model when surgical provider information was added, compared with only the variable for hospital of readmission. 
We also did several sensitivity analyses to evaluate the effect of hospital destination on mortality under various conditions. This analysis included stratification by emergency department admissions, hospital teaching status, hospital procedure volume level (low $\boldsymbol{v} \boldsymbol{s}$ high), and distance to the index hospital greater than 50 miles. We selected 50 miles as a cutoff because it represented roughly $1 \mathrm{~h}$ of travel time to the hospital. Dartmouth Human Investigation Committee deemed this study to be exempt from review.

\section{Role of the funding source}

There was no funding source for this study. BSB and PPG had full access to all the data in the study and BSB, PPG, and SRGF had final responsibility for the decision to submit for publication.

\section{Results}

We identified 9440503 patients during the study period who underwent one of 12 major operations within five surgical specialties (table 1). Across all procedures, prevalence of 30 day readmission ranged between 154203 (5.6\%) of 2748519 patients for knee replacement and 3665 (21.9\%) of 16702 patients for oesophagectomy.

In patients who needed to be readmitted to hospital within 30 days after major surgery, the number who were readmitted or transferred to the index hospital varied between procedures, from $186336(65.8 \%)$ of 283131 patients who were readmitted after coronary artery bypass grafting, to 142142 (83.2\%) of 170789 patients who were readmitted after colectomy (table 1). We calculated hospital-level index readmission prevalence within hospital referral regions for all 12 procedures, stratified by quartiles (appendix). The demographics and discharge destinations of patients who returned to the index hospital varied with the type of surgery (tables 2,3 ). Patients who were readmitted to the index hospital were significantly more likely to live in urban areas and to have travelled fewer miles to have their operation at a hospital within their same hospital referral region than were those who were admitted to a different hospital (tables 2, 3). Additionally, patients returning to index hospitals were readmitted within fewer days than were those who went to other hospitals.

We compared the characteristics of the hospitals that patients were readmitted to, stratified by whether these hospitals were the index hospital or non-index hospitals (tables 4, 5).

Generally, the index hospitals were smaller, with fewer staff and beds than were non-index hospitals (tables 4, 5). Moreover, index hospitals were more likely than non-index hospitals to be non-teaching hospitals, have lower SCIP-9 compliance, and be located in regions with lower mean incomes for the most surgical specialties (tables 4, 5).

The likelihood of patients being readmitted to the index hospital was increased when the readmission was to manage surgical complications compared with medical complications (tables 4, 5). Of the readmissions for medical complications, cardiac and infectious complications were most common overall. However, readmissions for medical or surgical complications were less likely to be for urgent or emergent indications if the patient returned to the index hospital than if they went to a non-index hospital (tables 4, 5). 
We collected crude 90 day mortality data for patients who were readmitted to index and nonindex hospitals for medical and surgical causes (appendix). For all types of surgery, unadjusted 90 day mortality was significantly lower for patients who were readmitted to the index hospital where surgery occurred than for patients readmitted to other hospitals (tables 4,5 , figure 1 ). These findings were supported by risk-adjusted, inverse probability weighted models for all 12 surgical procedures (index hospital readmission $\boldsymbol{v s}$ non-index hospital readmission, overall OR $0 \cdot 74,95 \%$ CI 0.66-0.83; figure 2). For all surgical procedures, 90 day mortality was reduced for patients readmitted to index hospitals compared with those admitted to non-index hospitals, and the effect was largest for those who underwent pancreatectomy $(0.56,0.45-0.69)$, aortobifemoral bypass $(0.69,0.61-0.77)$, colectomy $(0.75,0.73-0.77)$, and ventral hernia repair $(0.75,0.69-0.81$, figure 2$)$. Furthermore, readmission to the index hospital was the most consistent predictor of survival relative to the 74 other covariates in the model (appendix). We also identified similar results using logistic regression models without inverse probability weighting (data not shown).

In our instrumental variable analysis, we identified a similar, but attenuated, reduction in mortality for patients readmitted to the index hospital compared with those readmitted to non-index hospitals (figure 2). Patients with a higher probability of being readmitted to the index hospital instead of a non-index hospital after surgery had an $8 \%$ lower risk of 90 day mortality (overall OR $0 \cdot 92,95 \%$ CI $0 \cdot 91-0.94$ ) than did patients with a lower probability of index hospital readmission. For all 12 procedures assessed with instrumental variable analyses, ORs for risk-adjusted mortality favoured patients who returned to the index hospital, but this difference was significant for only six of 12 surgical procedures (figure 2).

Compared with readmission to non-index hospitals, the reduction in mortality was greatest when patients were readmitted to the index hospital for surgical complications (adjusted OR $0 \cdot 75,95 \%$ CI 0.74-0.77), and this effect existed for all surgical specialties (figure 1).

Furthermore, patients who needed management for a surgical complication at the time of readmission had a significant reduction in 90 day mortality in all comparisons if the same surgeon was involved in both the index and readmission treatment (figure 3). Knowledge of whether patients received care from the same surgical providers during readmission to the index hospital increased the $\mathrm{R}^{2}$ value by $2.9 \%$ relative to models with hospital of readmission alone, supporting an incremental benefit for maintenance of continuity with respect to treatment providers. Patients who were readmitted to the index hospital for medical complications also had significantly reduced risk of 90-day mortality (adjusted OR $0 \cdot 84,95 \%$ CI 0.83-0.85) compared with those readmitted to different hospitals, although this effect was reduced in comparisons of readmissions for surgical complications.

To determine whether the effect seen at 90 days existed earlier in the readmission process, we also examined in-hospital mortality during the readmission period for medical and surgical complications (appendix). We noted that in-hospital mortality was also significantly reduced for patients readmitted to index hospitals after all surgical procedures except for cardiothoracic operations (tables 4, 5). These results were supported by risk-adjusted weighted models: the only patients not to have a lower risk of in-hospital mortality associated with readmission to the index hospital were those readmitted after coronary artery bypass grafting (appendix). Additionally, we detected a similar in-hospital mortality benefit 
for patients who were readmitted under the care of the same provider for the management of surgical complications as for the index surgery (figure 3 ).

We did sensitivity analyses to examine the effect of hospital teaching status, distance greater than 50 miles to the index hospital, readmission through the emergency department, and volume of procedures at the hospital on risk-adjusted 90 day mortality models. The mortality benefit associated with readmission to the index hospital remained significant for all surgical procedures in these models, except for two procedures (open abdominal aortic aneurysm repair and ventral hernia repair) in patients who lived more than 50 miles from the index hospital (table 6).

\section{Discussion}

Patients undergoing major surgical procedures are often readmitted to hospitals to manage various medical and surgical complications, which are known to increase their risk of mortality. ${ }^{11}$ However, the best destination for these high-risk readmissions has not been established. Our results describe a consistent reduction in 90 day mortality for patients who were readmitted to the same hospital as where their surgery was done, for 12 diverse and common high-risk surgical procedures. In our inverse probability-weighted analysis, readmission to the index hospital was associated with a $26 \%$ reduction in risk of 90 day mortality compared with readmission to non-index hospitals. These results were supported by the hospital-level instrumental variable analysis, in which patients with the highest probability of index hospital readmission had an $8 \%$ lower risk of 90 day mortality than did patients with a lower likelihood of returning to the index hospital. This decrease in mortality risk was greatest for patients who were readmitted for surgical complications, rather than medical complications, especially when these patients were managed by the same surgical providers who did the index surgery. Together, these results suggest that patients who need readmission for complications after major surgery will have the best outcomes when managed by providers who maintain continuity of care throughout the patient's postoperative course.

Hospital readmissions after surgery have become a high-profile metric of health-care quality worldwide. ${ }^{8}$ Financial penalties for unplanned readmissions are now being enforced in the USA and the UK, with hospitals taking responsibility for readmissions, irrespective of whether patients return to the same hospital where the surgery was done or to another hospital. ${ }^{21-23}$ However, the association between readmission destination after surgery and patient outcomes has not been studied closely. A study ${ }^{24}$ that used a sample of $5 \%$ of Medicare claims for open and endovascular abdominal aortic aneurysm repair between 2005 and 2009 identified no benefit for 30 day mortality when patients returned to the same hospital where surgery was performed. ${ }^{24}$ These results contrast with those of our study, which used 100\% of Part A and B claims over a longer time period, and showed consistent reductions in in-hospital and 90 day mortality outcomes for 12 surgical procedures, including open abdominal aortic aneurysm repair. Furthermore, our main effect remained unchanged, even when our models controlled for established measures of surgical quality, such as hospital size, teaching status, and volume of procedures. The mortality reduction associated with index hospital readmission has face validity, which was further supported by 
our finding that this effect was most evident when the same surgical providers were involved in management of surgical complications (figure 3).

\section{Panel}

\section{Research in context}

\section{Systematic review}

Before we did this study, we searched PubMed and the Cochrane Library databases for all articles published between Jan 1, 1990, and Feb 1, 2014, that were relevant to hospital readmission destination after major surgery. We used the search terms "post-discharge", "continuity of patient care", "patient readmission", "operative procedures" and "surgery". Using these search criteria, we identified no prospective or retrospective studies that were applicable to this subject.

While the data for this study were being analysed, two relevant observational studies were published with some conflicting results. The first study assessed readmission destination after abdominal aortic aneurysm repair in a 5\% sample of US Medicare beneficiaries from 2005 to 2009 , but detected no significant mortality benefit associated with readmission to the index hospital. ${ }^{23}$ By contrast, another study that used $100 \%$ of Medicare claims for patients undergoing a composite of abdominal aortic aneurysm repair and four other procedures between 2009 and 2011 identified increased risk of 30 day mortality when patients were readmitted to hospitals other than where their surgery was performed. ${ }^{29}$ Although individual procedures or post-discharge complications were not assessed in this study, the results support an association between maintenance of continuity of post-discharge surgical care and improved survival.

\section{Interpretation}

Our data suggest that, when complications occur after major surgical procedures, patients who return to the index hospital and receive care from their original surgical team achieve significantly better 90 day survival than do patients whose readmission is to a non-index hospital. These data were consistent across a range of surgical procedures in models designed to control for measured and unmeasured confounding. Maintenance of continuity of post-discharge care within institutions where providers are familiar with a patient's surgical history should be regarded as a measure of surgical quality, and be considered carefully when patients select a hospital in which to undergo major surgery.

These findings raise important questions about the sustainability of worldwide health policies that aim to concentrate major or complex surgical procedures into specialised hospitals at the regional level. Patients increasingly travel long distances to have their operations done at hospitals that are recognised as providing high-quality care or because of a financial incentive for health insurers. ${ }^{25}$ This strategy has been adopted by several large corporations in the USA as a way to control spending on major surgical procedures by sending employees to hospitals that specialise in complex surgical care and accept bundled payments. ${ }^{26}$ Additionally, the Centers of Excellence programme, established by Centers of Medicare and Medicaid Services, will only pay for some high-risk surgical procedures that are done in approved facilities. ${ }^{27}$ These programmes make many patients travel to high- 
volume hospitals for their operations. When patients need readmission for complications, the assumption is that patients can seek care at local hospitals without a significant penalty in surgical outcomes. Our results challenge this theory, and we argue that continuity of surgical care needs to be treated as a competing metric of quality in choices of hospital in which to undergo major surgery.

Maintenance of continuity of care after hospital discharge has been shown to be a plausible and effective strategy to improve outcomes in a range of patients with high-risk medical disorders. ${ }^{15,16,28-30}$ For example, integrated post-hospital care delivery has been shown to reduce readmissions for patients with acute and chronic medical conditions, such as pneumonia, urinary tract infections, heart failure, and chronic obstructive pulmonary disease. ${ }^{15,16}$ Moreover, continuity of care has been reported to reduce complications and reduce overall health-care costs for patients with chronic diseases. ${ }^{15}$ This benefit is maintained when patients are cared for by teams within the same health-care setting, regardless of whether the same providers are involved with every episode of care. ${ }^{28}$ Although continuity of care in the management of patients undergoing surgery has not been thoroughly investigated, some studies ${ }^{12,29,30}$ suggest that patients returning to the same hospital and maintaining frequent contact with the surgical and primary care teams in the period following hospital discharge after high-risk surgery might have reduced risk of readmission and death. Our data further support the importance of continuity in surgical care, showing a dose-dependent reduction in mortality after readmission as the degree of continuity increased at the hospital level and provider level (figure 3). Moreover, our results suggest that continuity of care during readmission is a more consistent predictor of survival for patients who have undergone surgery after discharge from hospital than are other established quality measures such as hospital procedure volume.

Our study has several limitations. First, because our study was retrospective, readmission destination for patients was subject to selection bias and unmeasured confounding. This might include factors that determine severity of illness, time to presentation, and access to health care. Second, in our use of administrative billing data, we could not capture the full extent of the patient care continuity or conditions that determine medical complexity. Patients with the resources to return to the hospital where surgery was done might have clinical characteristics give a survival advantage. The findings from our instrumental variable analysis, however, reduce the likelihood that the effects seen for mortality result solely from unmeasured confounding. Third, our large, national study focused on Medicare patients and therefore our findings might not be generalisable to younger patient populations undergoing high-risk surgery or populations outside the USA.

Our results suggest that maintenance of continuity of surgical care is an important marker of quality, and should be taken into consideration in assessments of the advantages and potential unintended consequences of cost-effectiveness-driven regional centralisation of surgical care.

\section{Supplementary Material}

Refer to Web version on PubMed Central for supplementary material. 


\section{Acknowledgments}

This study was funded by BSB and SRGF. PPG was supported by a Career Development Award (K08 HL05676) from the National Heart Lung and Blood Institute.

\section{References}

1. Birkmeyer JD, Dimick JB, Birkmeyer NJ. Measuring the quality of surgical care: structure, process, or outcomes? J Am Coll Surg. 2004; 198:626-32. [PubMed: 15051016]

2. Ingraham AM, Richards KE, Hall BL, Ko CY. Quality improvement in surgery: the American College of Surgeons National Surgical Quality Improvement Program approach. Adv Surg. 2010; 44:251-67. [PubMed: 20919525]

3. Luft HS, Bunker JP, Enthoven AC. Should operations be regionalized? The empirical relation between surgical volume and mortality. N Engl J Med. 1979; 301:1364-69. [PubMed: 503167]

4. Hollenbeck BK, Miller DC, Wei JT, Montie JE. Regionalization of care: centralizing complex surgical procedures. Nat Clin Pract Urol. 2005; 2:461. [PubMed: 16474601]

5. Ghaferi AA, Birkmeyer JD, Dimick JB. Variation in hospital mortality associated with inpatient surgery. N Engl J Med. 2009; 361:1368-75. [PubMed: 19797283]

6. Ghaferi AA, Birkmeyer JD, Dimick JB. Complications, failure to rescue, and mortality with major inpatient surgery in medicare patients. Ann Surg. 2009; 250:1029-34. [PubMed: 19953723]

7. Hannan EL, O'Donnell JF, Kilburn H Jr, Bernard HR, Yazici A. Investigation of the relationship between volume and mortality for surgical procedures performed in New York State hospitals. JAMA. 1989; 262:503-10. [PubMed: 2491412]

8. Tsai TC, Joynt KE, Orav EJ, Gawande AA, Jha AK. Variation in surgical-readmission rates and quality of hospital care. N Engl J Med. 2013; 369:1134-42. [PubMed: 24047062]

9. Begg CB, Cramer LD, Hoskins WJ, Brennan MF. Impact of hospital volume on operative mortality for major cancer surgery. JAMA. 1998; 280:1747-51. [PubMed: 9842949]

10. Fry DE, Pine M, Pine G. Medicare post-discharge deaths and readmissions following elective surgery. Am J Surg. 2014; 207:326-30. [PubMed: 24418180]

11. Jencks SF, Williams MV, Coleman EA. Rehospitalizations among patients in the Medicare fee-forservice program. N Engl J Med. 2009; 360:1418-28. [PubMed: 19339721]

12. Brooke BS, Stone DH, Cronenwett JL, et al. Early primary care provider follow-up and readmissions after high-risk surgery. JAMA Surg. 2014; 149:821-28. [PubMed: 25074237]

13. Greenblatt DY, Weber SM, O'Connor ES, LoConte NK, Liou JI, Smith MA. Readmission after colectomy for cancer predicts one-year mortality. Ann Surg. 2010; 251:659-69. [PubMed: 20224370]

14. Greenblatt DY, Greenberg CC, Kind AJ, et al. Causes and implications of readmission after abdominal aortic aneurysm repair. Ann Surg. 2012; 256:595-605. [PubMed: 22964736]

15. Hussey PS, Schneider EC, Rudin RS, Fox DS, Lai J, Pollack CE. Continuity and the costs of care for chronic disease. JAMA Intern Med. 2014; 174:742-48. [PubMed: 24638880]

16. Nyweide DJ, Anthony DL, Bynum JP, et al. Continuity of care and the risk of preventable hospitalization in older adults. JAMA Intern Med. 2013; 173:1879-85. [PubMed: 24043127]

17. Romano PS, Roos LL, Jollis JG. Adapting a clinical comorbidity index for use with ICD-9-CM administrative data: differing perspectives. J Clin Epidemiol. 1993; 46:1075-79. [PubMed: 8410092]

18. Radley DC, Gottlieb DJ, Fisher ES, Tosteson AN. Comorbidity risk-adjustment strategies are comparable among persons with hip fracture. J Clin Epidemiol. 2008; 61:580-87. [PubMed: 18471662]

19. Stukel TA, Fisher ES, Wennberg DE, Alter DA, Gottlieb DJ, Vermeulen MJ. Analysis of observational studies in the presence of treatment selection bias: effects of invasive cardiac management on AMI survival using propensity score and instrumental variable methods. JAMA. 2007; 297:278-85. [PubMed: 17227979] 
20. Austin PC. An introduction to propensity score methods for reducing the effects of confounding in observational studies. Multivariate Behav Res. 2011; 46:399-424. [PubMed: 21818162]

21. Centers for Medicare and Medicaid Services. [accessed June 20, 2014] Readmissions reduction program. http://www.cms.gov/Medicare/Medicare-Fee-for-Service-Payment/AcuteInpatientPPS/ Readmissions-Reduction-Program.html

22. Fontanarosa PB, McNutt RA. Revisiting hospital readmissions. JAMA. 2013; 309:398-400. [PubMed: 23340644]

23. Santry C. Readmissions penalties could cost trusts $600 \mathrm{~m}$ pounds. Health Serv J. 2011; 121:13. [PubMed: 21882376]

24. Saunders RS, Fernandes-Taylor S, Kind AJ, et al. Rehospitalization to primary versus different facilities following abdominal aortic aneurysm repair. J Vasc Surg. 2014; 59:1502-10. [PubMed: 24491237]

25. Deloitte Center for Health Solutions. [accessed June 25, 2014] Medical tourism: consumers in search of value. http://www.deloitte.com/assets/Dcom-Croatia/Local\%20Assets/Documents/ hr_Medical_tourism.pdf

26. Wal-Mart, McKenzie K. [accessed June 28, 2014] Lowes use “corporate-sponsored medical tourism" to manage surgery bills. 2014. www.medcitynews.com/2014/02/wal-mart-lowes-usecorporate-sponsored-medical-tourism-manage-healthcare-costs/

27. Centers for Medicare and Medicaid Services. [accessed June 20, 2014] Medicare approved facilities. www.cms.gov/Medicare

28. Saultz JW. Defining and measuring interpersonal continuity of care. Ann Fam Med. 2003; 1:13443. [PubMed: 15043374]

29. Hall MH, Esposito RA, Pekmezaris R, et al. Cardiac surgery nurse practitioner home visits prevent coronary artery bypass graft readmissions. Ann Thorac Surg. 2014; 97:1488-93. [PubMed: 24612701]

30. Tsai TC, Orav EJ, Jha AK. Care fragmentation in the postdischarge period: surgical readmissions, distance of travel, and postoperative mortality. JAMA Surg. 2015; 150:59-64. [PubMed: 25472595] 


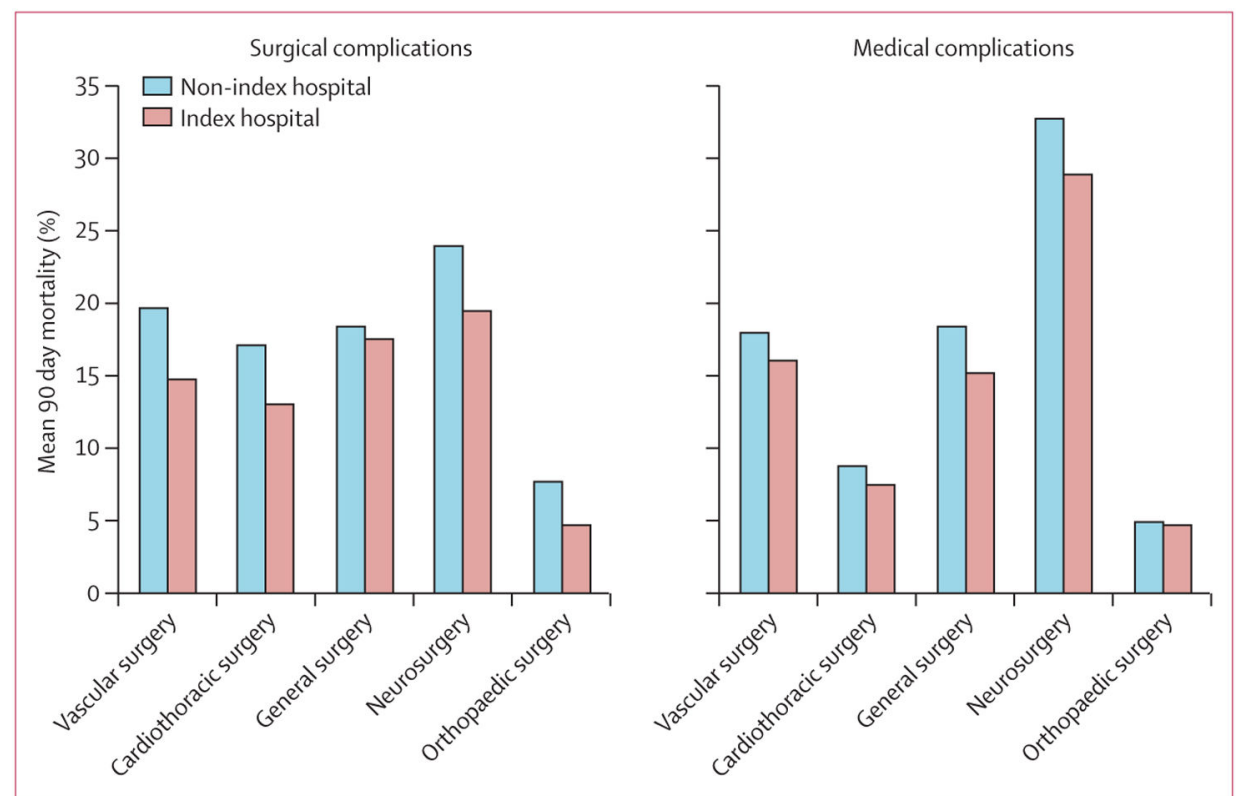

Figure 1. Crude 90 day mortality

Cardiothoracic surgery=coronary artery bypass surgery, oesophagectomy. General surgery=cholecystectomy, pancreatectomy, colectomy, ventral hernia repair. Neurosurgery=craniotomy. Orthopaedic surgery=hip replacement, knee replacement. 
Open abdominal aortic aneurysm repair Inverse probability weighting estimate Instrumental variable estimate

Aortobifemoral bypass

Inverse probability weighting estimate

Instrumental variable estimate

Infrainguinal arterial bypass

Inverse probability weighting estimate

Instrumental variable estimate

Coronary artery bypass surgery

Inverse probability weighting estimate

Instrumental variable estimate

Oesophagectomy

Inverse probability weighting estimate

Instrumental variable estimate

Cholecystectomy

Inverse probability weighting estimate

Instrumental variable estimate

Pancreatectomy

Inverse probability weighting estimate

Instrumental variable estimate

Colectomy

Inverse probability weighting estimate

Instrumental variable estimate

Ventral hernia repair

Inverse probability weighting estimate

Instrumental variable estimate

Craniotomy

Inverse probability weighting estimate

Instrumental variable estimate

Hip replacement

Inverse probability weighting estimate

Instrumental variable estimate

Knee replacement

Inverse probability weighting estimate

Instrumental variable estimate

Overall

Inverse probability weighting estimate

Instrumental variable estimate

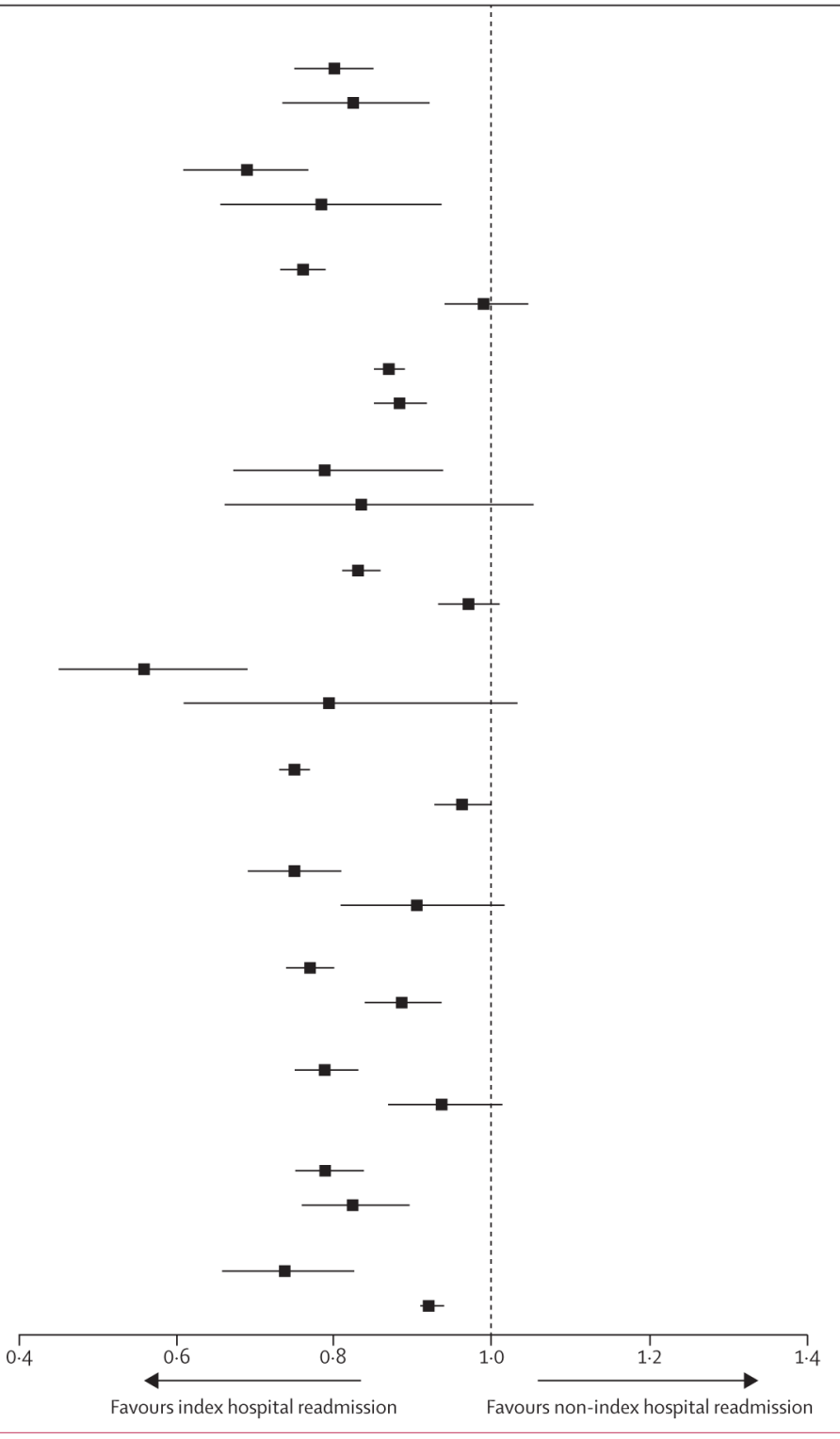

$0.80(0.75-0.85)$

$0.82(0.74-0.92)$

$0.69(0.61-0.77)$

$0.78(0.66-0.94)$

$0.76(0.73-0.79)$

$0.99(0.94-1.05)$

$0.87(0.85-0.89)$

$0.88(0.85-0.92)$

$0.79(0.67-0.94)$

$0.83(0.66-1.05)$

$0.83(0.81-0.86)$

$0.97(0.93-1.01)$

$0.56(0.45-0.69)$

$0.79(0.61-1.03)$

$0.75(0.73-0.77)$

$0.96(0.93-1.00)$

$0.75(0.69-0.81)$

$0.90(0.81-1.02)$

$0.77(0.74-0.80)$

$0.91(0.81-1.02)$

$0.79(0.75-0.84)$

$0.94(0.87-1.01)$

$0.79(0.75-0.84)$

$0.82(0.76-0.89)$

$0.74(0.66-0.83)$

$0.92(0.91-0.94)$

Figure 2.

Inverse probability weighting and instrumental variable analyses of 90 day mortality

Lancet. Author manuscript; available in PMC 2016 April 29. 


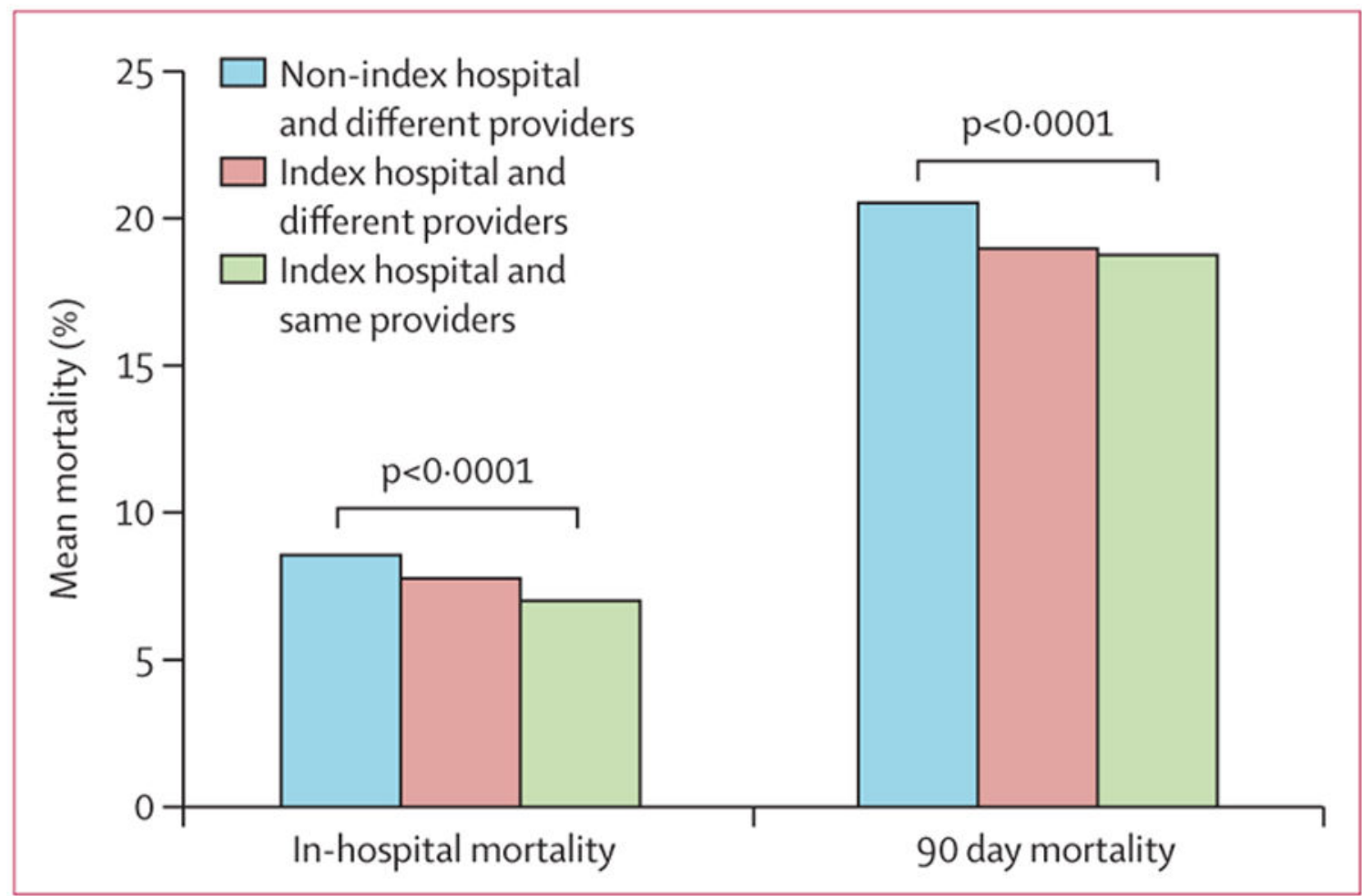

Figure 3. Effects of continuity of care on in-hospital mortality and 90 day mortality Data stratified by whether patients returned to the index hospital where surgery occurred and whether they were managed by the same or different providers during index and readmission hospital stays. 


\section{Table 1}

Patients readmitted after 12 major operations in five surgical specialties

\begin{tabular}{|c|c|c|c|}
\hline & $\begin{array}{r}\text { Patients discharged after } \\
\text { surgery }\end{array}$ & $\begin{array}{r}\text { Patients readmitted to any } \\
\text { hospital }\end{array}$ & $\begin{array}{l}\text { Patients readmitted who were } \\
\text { readmitted to index hospital }\end{array}$ \\
\hline \multicolumn{4}{|l|}{ Vascular surgery } \\
\hline Open abdominal aortic aneurysm repair & 163753 & $26002(15 \cdot 9 \%)$ & $18220(70 \cdot 1 \%)$ \\
\hline Aortobifemoral bypass & 67826 & $11498(17 \cdot 0 \%)$ & $8739(76.0 \%)$ \\
\hline Infrainguinal arterial bypass & 448296 & $90596(20 \cdot 2 \%)$ & $72143(79 \cdot 6 \%)$ \\
\hline \multicolumn{4}{|l|}{ Cardiothoracic surgery } \\
\hline Coronary artery bypass surgery & 1502815 & $283131(18.8 \%)$ & $186336(65 \cdot 8 \%)$ \\
\hline Oesophagectomy & 16702 & $3665(21.9 \%)$ & $2447(66.8 \%)$ \\
\hline \multicolumn{4}{|l|}{ General surgery } \\
\hline Cholecystectomy & 1435157 & $183494(12.7 \%)$ & $148520(80 \cdot 9 \%)$ \\
\hline Pancreatectomy & 16778 & $3582(21 \cdot 3 \%)$ & $2670(74.5 \%)$ \\
\hline Colon resection & 1110967 & $170789(15.4 \%)$ & $142142(83 \cdot 2 \%)$ \\
\hline Ventral hernia repair & 302196 & $38958(12.9 \%)$ & $32248(82.8 \%)$ \\
\hline \multicolumn{4}{|l|}{ Neurosurgery } \\
\hline Craniotomy & 355075 & $55974(15 \cdot 8 \%)$ & $39195(70 \cdot 0 \%)$ \\
\hline \multicolumn{4}{|l|}{ Orthopaedic surgery } \\
\hline Hip replacement & 1272419 & $89154(7 \cdot 0 \%)$ & $68069(76 \cdot 3 \%)$ \\
\hline Knee replacement & 2748519 & $154203(5 \cdot 6 \%)$ & $113335(73 \cdot 5 \%)$ \\
\hline
\end{tabular}




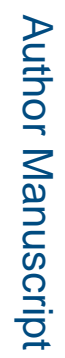

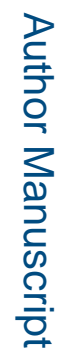

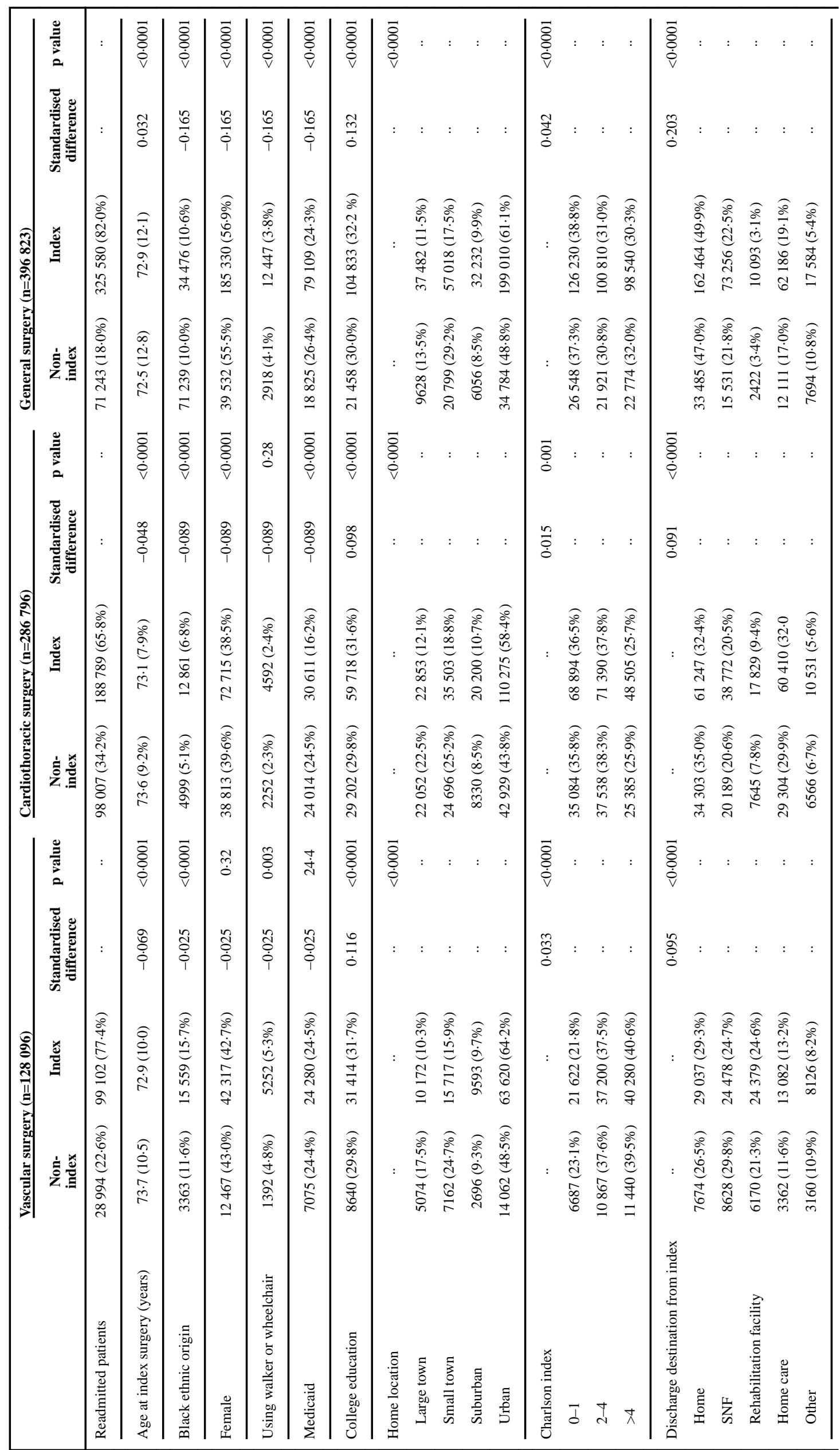




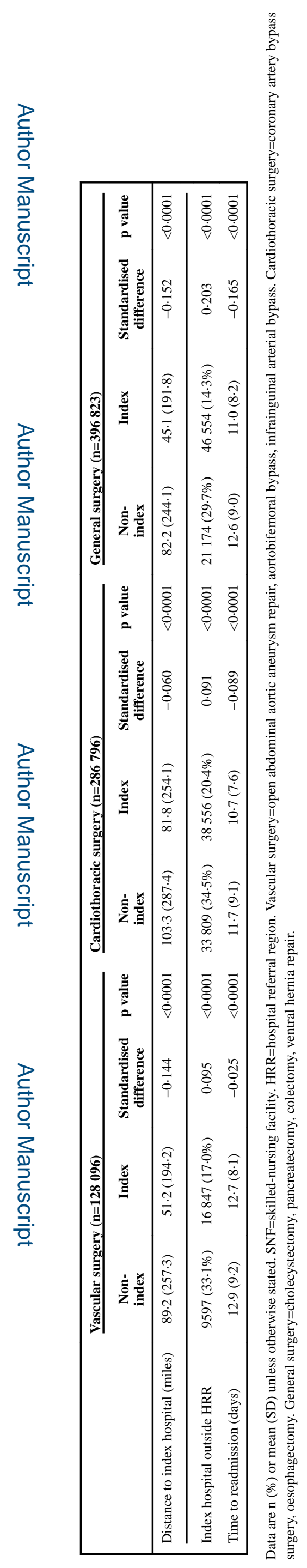


롤 


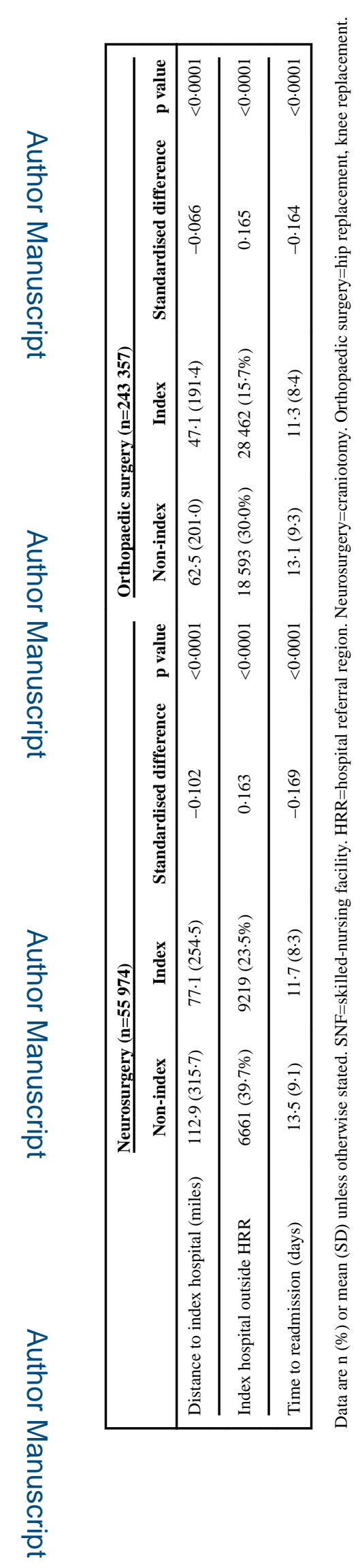




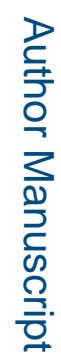

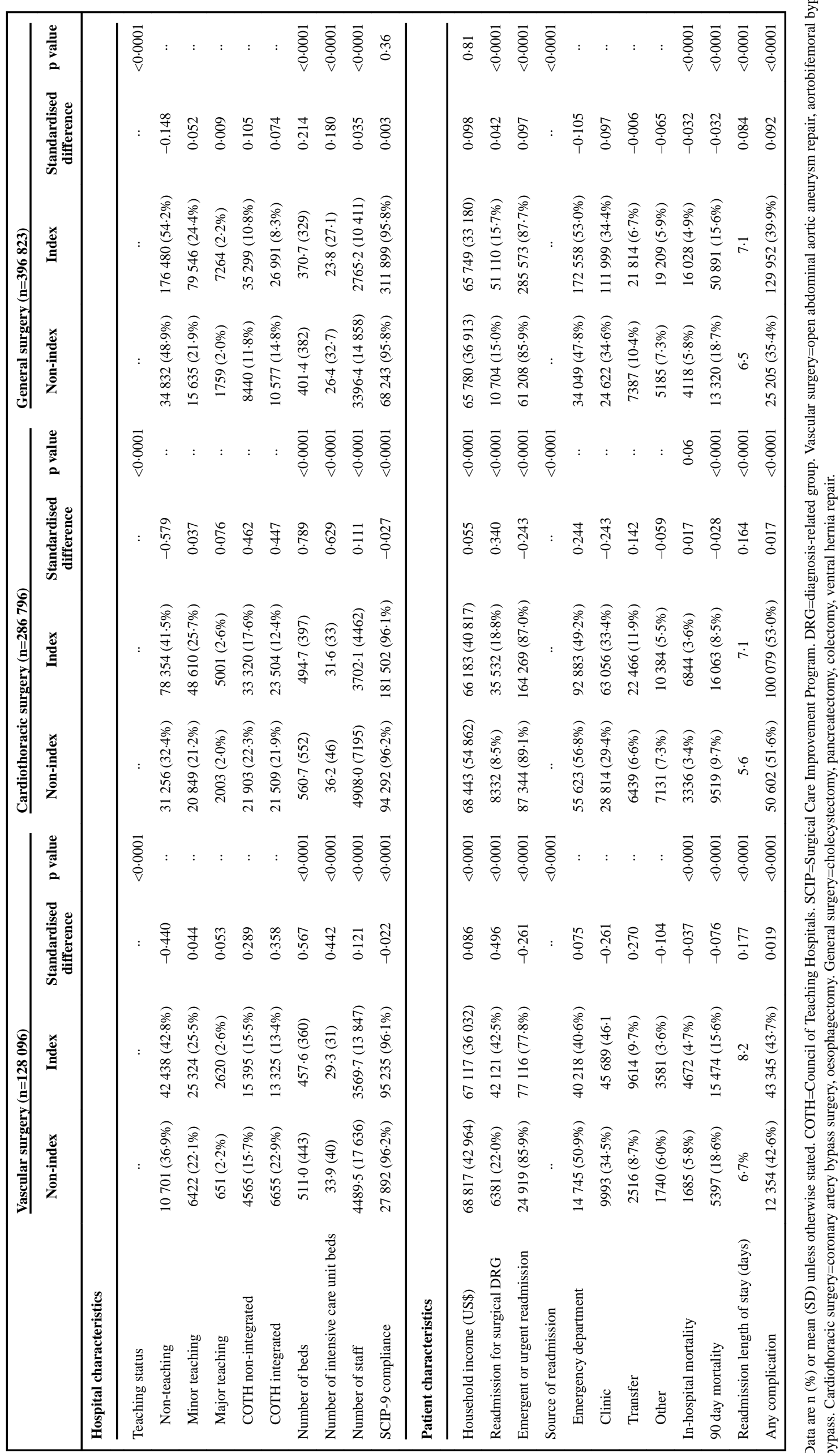




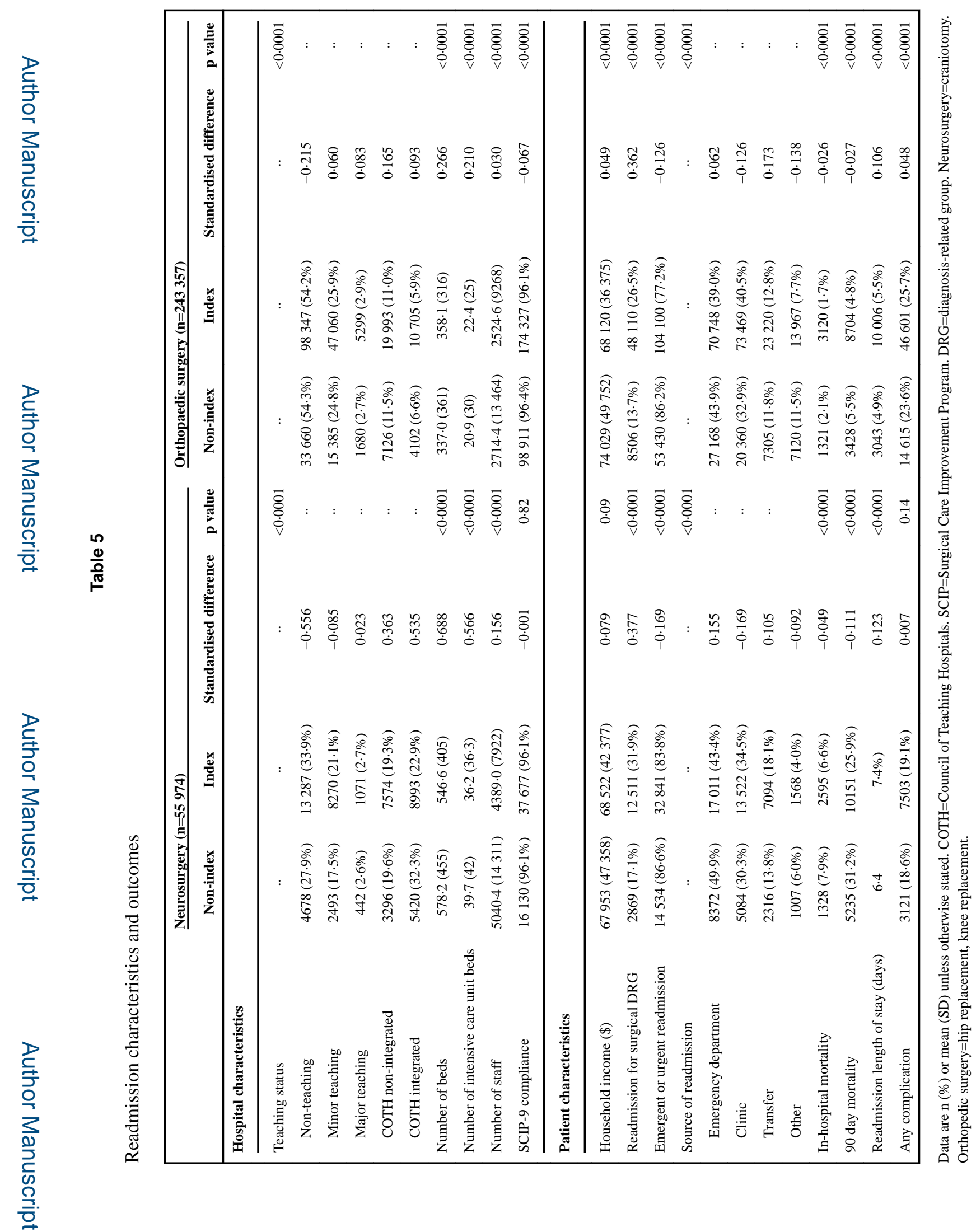

Lancet. Author manuscript; available in PMC 2016 April 29. 
Table 6

Sensitivity analysis

\begin{tabular}{|c|c|c|c|}
\hline & Odds ratio & $95 \% \mathrm{CI}$ & p value \\
\hline \multicolumn{4}{|l|}{ Major teaching hospital } \\
\hline Open abdominal aortic aneurysm repair & $0 \cdot 68$ & $0 \cdot 58-0 \cdot 80$ & $<0.0001$ \\
\hline Aortobifemoral bypass & $0 \cdot 31$ & $0 \cdot 23-0 \cdot 42$ & $<0.0001$ \\
\hline Infrainguinal arterial bypass & $0 \cdot 64$ & $0.58-0 \cdot 71$ & $<0.0001$ \\
\hline Coronary artery bypass surgery & 0.59 & $0.56-0 \cdot 63$ & $<0.0001$ \\
\hline Cholecystectomy & 0.73 & $0.67-0.79$ & $<0.0001$ \\
\hline Colectomy & $0 \cdot 68$ & $0 \cdot 63-0.73$ & $<0.0001$ \\
\hline Ventral hernia repair & 0.52 & $0 \cdot 42-0 \cdot 65$ & $<0.0001$ \\
\hline Craniotomy & 0.79 & $0 \cdot 73-0 \cdot 86$ & $<0.0001$ \\
\hline Hip replacement & $0 \cdot 64$ & $0.56-0 \cdot 73$ & $<0.0001$ \\
\hline \multicolumn{4}{|l|}{ Non-teaching hospital } \\
\hline Open abdominal aortic aneurysm repair & $0 \cdot 85$ & $0 \cdot 72-0 \cdot 85$ & $<0.0001$ \\
\hline Aortobifemoral bypass & $0 \cdot 68$ & $0.59-0 \cdot 78$ & $<0.0001$ \\
\hline Infrainguinal arterial bypass & 0.79 & $0 \cdot 76-0 \cdot 83$ & $<0.0001$ \\
\hline Coronary artery bypass surgery & $0 \cdot 87$ & $0 \cdot 84-0 \cdot 89$ & $<0.0001$ \\
\hline Cholecystectomy & $0 \cdot 84$ & $0 \cdot 81-0 \cdot 87$ & $<0.0001$ \\
\hline Colectomy & $0 \cdot 76$ & $0.74-0.79$ & $<0 \cdot 0001$ \\
\hline Ventral hernia repair & 0.75 & $0 \cdot 68-0 \cdot 82$ & $<0.0001$ \\
\hline Craniotomy & $0 \cdot 78$ & $0 \cdot 74-0 \cdot 81$ & $<0.0001$ \\
\hline Hip replacement & $0 \cdot 80$ & $0 \cdot 75-0 \cdot 85$ & $<0.0001$ \\
\hline \multicolumn{4}{|l|}{ Distance $<\mathbf{5 0}$ miles to index hospital } \\
\hline Open abdominal aortic aneurysm repair & $0 \cdot 76$ & $0 \cdot 71-0 \cdot 83$ & $<0 \cdot 0001$ \\
\hline Aortobifemoral bypass & $0 \cdot 65$ & $0.57-0.75$ & $<0.0001$ \\
\hline Infrainguinal arterial bypass & $0 \cdot 80$ & $0 \cdot 77-0 \cdot 84$ & $<0.0001$ \\
\hline Coronary artery bypass surgery & $0 \cdot 81$ & $0.79-0.83$ & $<0.0001$ \\
\hline Cholecystectomy & $0 \cdot 82$ & $0 \cdot 79-0 \cdot 84$ & $<0.0001$ \\
\hline Colectomy & 0.73 & $0 \cdot 70-0 \cdot 75$ & $<0.0001$ \\
\hline Ventral hernia repair & $0 \cdot 70$ & $0.64-0.76$ & $<0.0001$ \\
\hline Craniotomy & 0.77 & $0 \cdot 74-0 \cdot 81$ & $<0.0001$ \\
\hline Hip replacement & $0 \cdot 81$ & $0 \cdot 76-0 \cdot 85$ & $<0.0001$ \\
\hline \multicolumn{4}{|l|}{ Distance $\Sigma 0$ miles to index hospital } \\
\hline Open abdominal aortic aneurysm repair & $0 \cdot 93$ & $0 \cdot 83-1 \cdot 05$ & $0 \cdot 26$ \\
\hline Aortobifemoral bypass & $0 \cdot 68$ & $0 \cdot 54-0 \cdot 86$ & $0 \cdot 002$ \\
\hline Infrainguinal arterial bypass & 0.72 & $0 \cdot 65-0 \cdot 78$ & $<0.0001$ \\
\hline Coronary artery bypass surgery & 1.05 & $1 \cdot 01-1 \cdot 09$ & $0 \cdot 03$ \\
\hline Cholecystectomy & $0 \cdot 88$ & $0 \cdot 82-0 \cdot 94$ & $<0.0001$ \\
\hline
\end{tabular}




\begin{tabular}{|lccc|}
\hline & Odds ratio & 95\% CI & p value \\
\hline Colectomy & 0.86 & $0.81-0.92$ & $<0.0001$ \\
Ventral hernia repair & 0.87 & $0.72-1.04$ & 0.12 \\
Craniotomy & 0.73 & $0.68-0.78$ & $<0.0001$ \\
Hip replacement & 0.77 & $0.67-0.88$ & $<0.0001$ \\
\hline
\end{tabular}

Risk-adjusted odds ratio of 90 day mortality for readmission to same hospital stratified by teaching status (major teaching $v s$ non-teaching) and distance to index hospital ( $<50$ miles $v s \geq 50$ miles). The reference group for all sensitivity analyses is readmission to other hospitals than where surgery occurred. We only included data for the nine procedures for which stratified regression models were able to converge. 